First publ. in: Physica Status Solidi / A, Vol. 166 (1998), 2, S. 643-650

\title{
On the Interpretation of Time-Resolved Surface Reflectivity Measurements during the Laser Annealing of Si Thin Films
}

\author{
J. BoneberG ${ }^{1}$ ) and P. Leiderer \\ Fakultät für Physik, Universität Konstanz, D-78457 Konstanz, Germany
}

(Received February 20, 1998)

\begin{abstract}
Time-resolved surface reflectivity measurements of nanosecond pulse laser-annealed Si thin films are compared to the simultaneously measured reflectivity of the interface between the film and glass substrate. In the case of complete melting of the film the liquid is supercooled. The measurements of the interface reflectivity show that the resolidification process starts at the interface. The resolidification at the surface occurs after an energy-dependent delay of up to $40 \mathrm{~ns}$. The analysis of the data shows that during this delay time the surface temperature may be increased by the latent heat released at the interface. This reheating effect of the surface, which has not been considered yet demands for a careful interpretation of surface reflectivity measurements.
\end{abstract}

\section{Introduction}

Laser annealing of $\mathrm{Si}$ was a subject of numerous studies during the 80ies, triggered especially by the question of a possible laser recrystallization of ion-implanted surface layers [1 to 3]. In these investigations many important questions were adressed, as e.g. the appearance of explosive recrystallization. Time-resolved reflectivity (TRR) studies were established as an important tool to monitor the dynamics at surfaces or interfaces. In the last few years a revival of the laser-annealing technique could be observed, which was especially applied for the crystallization of thin amorphous Si films intended for the use in thin film transistors [4 to 7]. For that reason also TRR is increasingly used. Although the technique is simple in principle, the interpretation is not as straightforward as long as no special care is taken of phase changes, temperature changes and interference effects, which may appear simultaneously. We focus here on an additional effect which is important for the interpretation of the surface TRR in thin films. It will be shown that the latent heat released by the resolidification at the interface dominates the surface resolidification. Therefore, the TRR studies at the surface may on a first view arise unexpected results.

\section{Experimental}

The annealing laser was a frequency-doubled $Q$-switched Nd:YAG laser with a pulse length of $7 \mathrm{~ns}$ (full width at half maximum) and a beam profile close to $\mathrm{TEM}_{00}$. The $\mathrm{Nd}$ :YAG pulse incidents nearly perpendicular to the surface (Fig. 1), and is only 
mildly focused to a spot diameter of about $0.5 \mathrm{~mm}$. For the measurement of the reflectivity we used two p-polarized continuous wave (cw) lasers: one of them (a $\mathrm{He}-\mathrm{Ne}$ laser at $633 \mathrm{~nm}$, angle of incidence $45^{\circ}$ ) tested the reflectivity at the surface, whereas an argon ion laser $\left(488 \mathrm{~nm}\right.$, angle of incidence $35^{\circ}$ ) was used to monitor the reflectivity at the interface between film and substrate. Both lasers were focussed to a $1 / e$ diameter of below $15 \mu \mathrm{m}$ at the surface and interface respectively, so that the variation of the pulse laser beam intensity across the diameter of the test laser could be neglected. The overlap of the test laser foci was better than $90 \%$. The specularly reflected or transmitted light was detected by pin diodes and registered by a fast digital storage oscilloscope (HP54111D). The time resolution of the measurement was $1 \mathrm{~ns}$. Interference filters in front of the pin diodes suppressed any contribution of the pulse laser to the measured signal. Our samples were $125 \mathrm{~nm}$ polycrystalline Si thin films on quartz glass substrates.

\section{Results and Discussion}

The experimental results can be divided into three regimes, depending on the energy density of the annealing laser: heating of the solid Si film, partial melting and complete melting of the Si film.

\subsection{Heating of the Si film}

As long as the energy density of the annealing laser pulse is sufficiently low $\left(E<E_{\mathrm{m}}\right.$ $=170 \mathrm{~mJ} / \mathrm{cm}^{2}$ ) the film does not melt. Nevertheless, variations of the reflectivity of about $20 \%$ are observed. These arise from the temperature dependence of the optical constants of crystalline Si [8] and have been shown elsewhere [9]. The absolute changes are less pronounced for the wavelength $488 \mathrm{~nm}$ as the absorption at this wavelength is higher, which reduces interference effects.

\subsection{Partial melting of the Si film}

As the energy density is increased above $E_{\mathrm{m}}$, a surface layer of $\mathrm{Si}$ is melted and consequently additional structures to the interference effects in $R_{\mathrm{S}}$ and $R_{\mathrm{I}}$ appear. As long as the liquid layer is present at the surface with a thickness $d>2 d_{\mathrm{ab}}$ (where $d_{\mathrm{ab}}$ is the absorption length in liquid $\mathrm{Si}$, about $10 \mathrm{~nm}$ [10]) $R_{\mathrm{S}}$ remains nearly constant and thus a plateau appears (Fig. 1). The interface reflectivity $R_{\mathrm{I}}$, on the other hand, does not show a plateau: the liquid-solid boundary moves towards the interface as long as the energy input exceeds the heat transport into the substrate. At the end of the laser pulse the energy balance is reversed and thus the interface moves in the other direction. The transition from melting towards resolidification some nanometer apart from the interface leads to a pronounced peak in $R_{\mathrm{I}}$. The recrystallization proceeds from the region of the interface towards the surface. The time delay between resolidification near the interface and surface resolidification amounts to about $40 \mathrm{~ns}$.

\subsection{Complete melting of the Si film}

In the case of complete melting a change of the resolidification scenario is expected [11 to 13]: Due to the lack of nucleation sites the liquid can be undercooled until at suffi- 


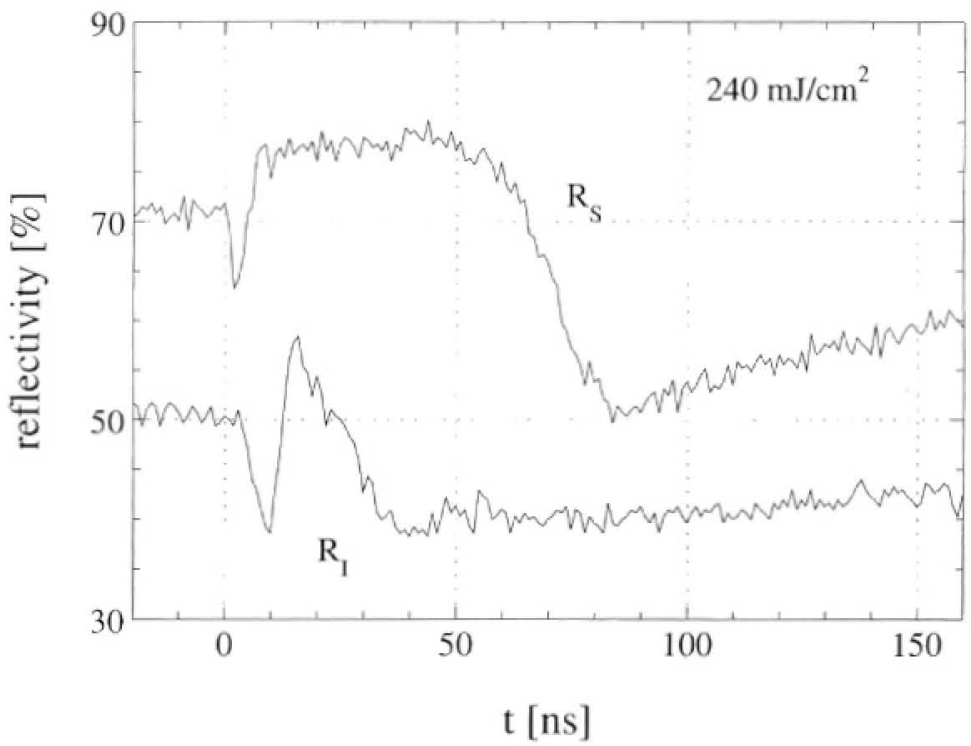

Fig. 1. Surface reflectivity $R_{S}$ and interface reflectivity $R_{1}$ for an energy density of $240 \mathrm{~mJ} / \mathrm{cm}^{2}$

cient supercooling nucleation sites are formed, which then grow rapidly. As a result the latent heat is released which increases the temperature of the film up to the melting point. This scenario is strongly supported by the measurement of $R_{\mathrm{S}}$ at higher energy densities (Fig. 2). After the first plateau which indicates the supercooling of the liquid $\mathrm{Si}$ a fast decrease of $R_{\mathrm{S}}$ appears which can be attributed to a solidification process. The

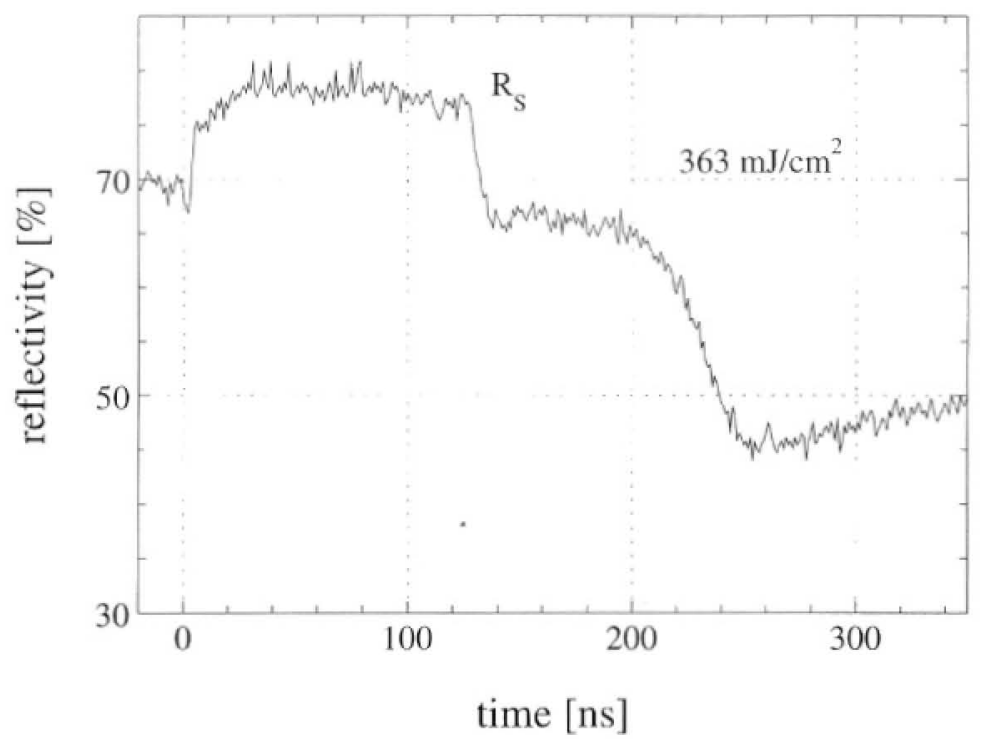

Fig. 2. Surface reflectivity $R_{\mathrm{S}}$ for an energy density of $363 \mathrm{~mJ} / \mathrm{cm}^{2}$ 
temperature raises towards the melting point and further growth in the liquid-solid mixture is stopped, giving rise to a second plateau in $R_{\mathrm{S}}$ with a reflectivity value of this mixture before complete solidification takes place.

The model sketched above has important consequences for the reflectivity of the second plateau. The appearance of a plateau indicates that no further resolidification process (or at least only very slow resolidification) takes place within a thickness given by the penetration depth of the test laser. Since nucleation sites are available at this moment it follows that the temperature of the liquid-solid mixture has to be close to the melting point. The heat required for the temperature increase from the nucleation temperature back to the melting temperature has to be supplied by the latent heat of resolidification. Thus, for a liquid with higher supercooling more energy is necessary to reach the melting temperature. This transforms into a higher degree of resolidification and consequently a smaller reflectivity in the second plateau.

Higher supercooling is experimentally realized by decreasing the energy density in the range of complete melting. In Fig. $3, R_{\mathrm{S}}$ of the second plateau is shown for the complete range of energy densities. For comparison, the values for the liquid phase and for a $60 \%$ solid/40\% liquid mixture as calculated by the Bruggemann approach are given. The absolute change of $R_{\mathrm{S}}$ is only on the order of a few percent. In contrast to the behaviour expected on the basis of the model the reflectivity first grows with increasing supercooling and then drops again. From this discrepancy one can conclude that the model has to be modified. The main aspect that has to be considered is the dynamic behaviour of the resolidification across the film. Even though, calculations predict a small temperature gradient in the film, in our measurements the nucleation always started at the interface, as will be shown in the following.

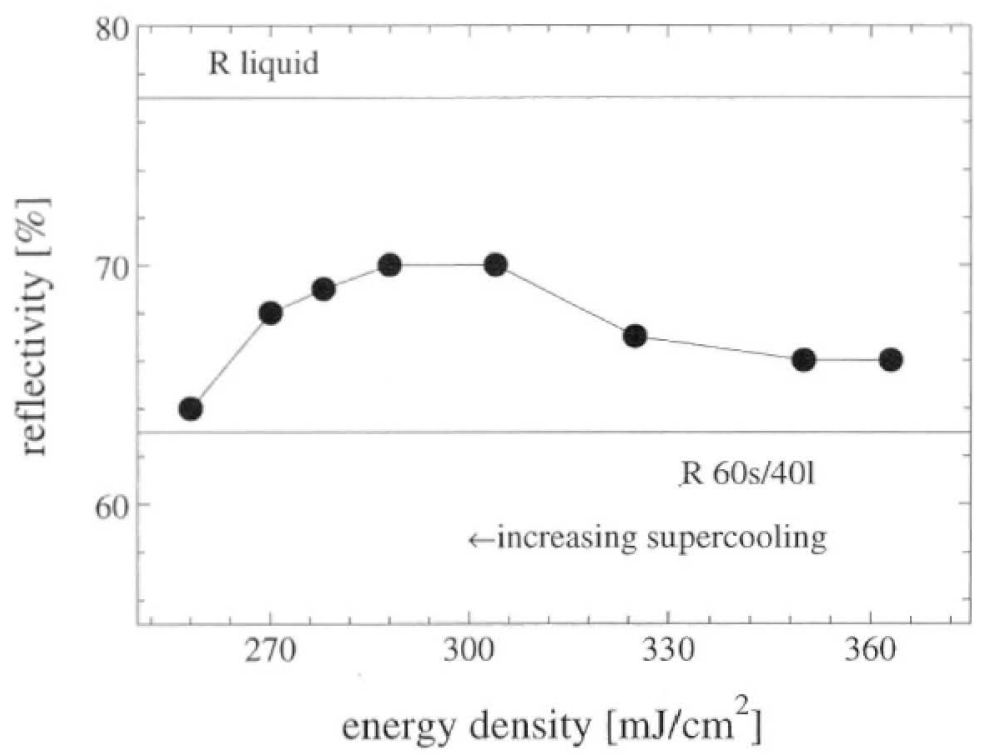

Fig. 3. Surface reflectivity $R_{\mathrm{S}}$ of the second plateau as function of energy density. The calculated values for a pure liquid and a $60 \%$ solid $/ 40 \%$ liquid mixture are given as well 


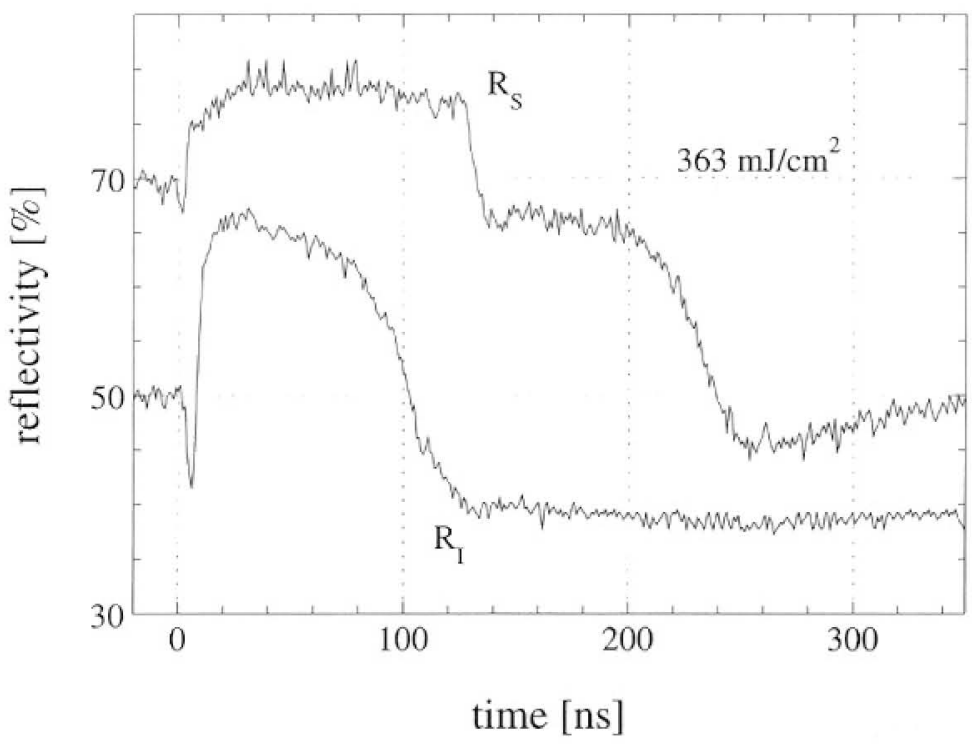

Fig. 4. Surface reflectivity $R_{\mathrm{S}}$ and interface reflectivity $R_{1}$ for an energy density of $363 \mathrm{~mJ} / \mathrm{cm}^{2}$

Fig. 4 presents a comparison of $R_{\mathrm{S}}$ and $R_{\mathrm{I}}$ for the energy density $360 \mathrm{~mJ} / \mathrm{cm}^{2}$. It is obvious that the behaviour of $R_{\mathrm{I}}$ is quite different. In particular no second plateau is found. This corresponds to a complete solidification in one step. Most important for the discussion here is the delay between the resolidification at the surface and the interface. Although the plateau in $R_{\mathrm{I}}$ does not show a constant reflectivity (we assume that this is due to the change of the optical properties of the quartz glass substrate) it is possible to determine the beginning of resolidification from the change of the slope of $R_{\mathrm{I}}$ to around $t=85 \mathrm{~ns}$. The first change of the surface reflectivity, on the other hand, appears $43 \mathrm{~ns}$ later, when the resolidification at the interface is already completed. This implies that latent heat was released at a layer near the interface, whereas the surface is still in a supercooled state. Thus the temperature gradient in the film may be reversed, which implies that it becomes difficult or even impossible to determine the temperature at the surface as long as the exact resolidification rate (which itself is a function of the local temperature) throughout the film is not known.

The delay between the beginning of interface and surface resolidification depends on the energy density as shown in Fig. 5. For increasing supercooling the time delay is reduced. The minimum time delay amounts to $8 \mathrm{~ns}$, which is quite large compared to the time which is necessary for heat diffusion throughout the film (below $1 \mathrm{~ns}$ ).

These results show that the interpretation of the surface reflectivity data is difficult, as the surface effects seem to be dominated by the interface history. A further test of this assumption is the analysis of the regrowth velocity from the liquid supercooled state, corresponding to the change of reflectivity after the first plateau. As already stated the resolidification velocity after the formation of nuclei should reflect the magnitude of supercooling. Assuming the linear change of reflectivity as function of the phase change from liquid to the solid state, $\mathrm{d} R / \mathrm{d} t$ can be considered to be proportional to the resolidification velocity. The data show once again the quoted beha- 
[6] M. Heintze, P. V. Santos, C. E. Nebel, and M. Stutzmann, Appl. Phys. Lett. 64, 3184 (1994).

[7] R. Ishihara and M. Matsumura, Jpn. J. Appl. Phys. 34, 3976 (1995).

[8] G. E. Jellison, Jr. and H. H. Burke, J. Appl. Phys, 60, 841 (1986).

[9] J. Boneberg, J. Nedelcu, H. Bender, and P. Leiderer, Mater. Sci. Engng. A 173, 347 (1993).

[10] G. E. Jellison, Jr., D. H. Lowndes, D. N. Mashburn, and R. F. Wood, Phys. Rev. B 34,2407 (1986).

[11] S. R. Stiffler, M. O. Thompson, and P. S. Peercy, Phys. Rev. B 43, 9851 (1991).

[12] S. R. Stiffler, M. O. Thompson, and P. S. Peercy, Phys. Rev. Lett. 60, 2519 (1988).

[13] S. R. Stiffler, Dissertation, Cornell University, 1988. 